\title{
We Asked the Experts: The Promises and Challenges of Surgical Telehealth in Low Resourced Settings
}

\author{
Phoebe Miller $^{1} \cdot$ Eyitayo Owolabi ${ }^{2} \cdot$ Kathryn $\mathrm{Chu}^{2,3}$ (D)
}

Access to safe and timely surgical care saves lives, but its multiple barriers in low- and middle-income countries (LMICs) contribute to high postoperative mortality [1]. In these settings, surgical health systems are fragile due to a shortage of supplies such as drugs, anesthesia equipment and oxygen, the maldistribution of surgical specialists, poor referral systems, and an inability to routinely track processes and outcomes indicators for quality improvement. The ongoing Covid-19 pandemic has heightened barriers to surgical care in LMICs with resultant increases in unmet surgical needs. On the other hand, the pandemic has revealed the great potentials of telehealth.

Telehealth, which is the provision of healthcare-related services over a distance using electronic and telecommunication technologies, has created solutions to leapfrog certain barriers to surgical care in LMICs. Long distance travel to reach facilities and extended waiting times to see specialists can be circumvented by phone and online consultations. These virtual visits are not only cost saving but can prevent critical delays in patient care. Remote consultations can take on various forms. Firstly, initial visits and preoperative instructions can be done through telehealth platforms from the comfort of patient homes. In certain low acuity and elective cases, video visits may

Kathryn Chu

kchu@sun.ac.za

1 School of Medicine, University of California-San Francisco, 1400 Parnassus Avenue, San Francisco, CA, USA

2 Centre for Global Surgery, Department of Global Health, Faculty of Medicine and Health Sciences, Stellenbosch University, Francie Van Zijl Drive, Tygerberg, Cape Town 7505, South Africa

3 Department of Surgery, University of Botswana, Plot 4775 Notwane Rd, Gaborone, Botswana make it possible to determine the need for an operation or the need for in-person visitation to assist surgical planning. Additionally, mobile apps, direct phone calls, and instant messaging are suitable for preoperative education and assisting patients in navigating barriers to surgical access in addition to using video chat platforms. Likewise, mHealth apps and real-time video features allow for postoperative follow-up including routine wound inspection and utilize community health workers, nurses, or general medical doctors located closer to the patient than the hospital that provided the surgical care. The addition of artificial intelligence technology to mHealth could aid these cadres to identify wound infections. In Rwanda, machine learning is being harnessed to detect postoperative wound infections in rural women after Cesarean sections [2]. Finally, outreach by surgeons to rural areas can be strengthened by remote preoperative consultations to identify appropriate operative candidates, provide virtual spaces for planning with local teams, and conduct postoperative follow-up. Therefore, telehealth maximizes the impact of visiting specialists and improves the quality of patient care.

Poor communication and referral networks between health facilities are major barriers to timely and quality access to surgical care in LMICs. Telehealth allows doctors and nurses in rural and primary care facilities to communicate quickly with surgeons at regional and tertiary hospitals. The mHealth app, Vula Mobile, is used ubiquitously by South African rural doctors and nurses to refer persons with surgical conditions to specialists at higher level hospitals. A 2019 study showed that one-third of acute orthopedic conditions were managed on this platform through expert advice without the need for transfer [3]. The median response time on the app was less than 30 minutes. In addition, metadata from mHealth referral apps can be used to track volumes, referral times, and patient flow, 
which might be used for quality improvement efforts. This type of telehealth platform shows promise and might be scaled-up in other LMICs to better link networks of nonspecialist health care providers and surgeons.

If higher bandwidth is available, real-time video platforms, which allow for in-depth consultations and case discussions, can be used to overcome specialist shortages in LMICs. Virtual multi-disciplinary conferences are being used in South-South and North-South collaborations. For example, the Global Cancer Institute has a network of over 500 doctors from Africa, Asia, and Latin America who present cancer cases for discussion with US oncology experts [4].

The limited case mix at some LMIC training hospitals and the shortage of surgical subspecialists can impede the acquisition of certain operative skills. Telesurgery, or intraoperative tele-mentoring, is where a senior surgeon located remotely can give immediate and continuous feedback to the operating surgeon. Early attempts at South-South telesurgery collaborations have shown good patient outcomes [5].

Another telehealth innovation for skills acquisition is simulation, or the use of models to imitate the steps of an operation. Simulators can be high-fidelity units with computer animation or low-fidelity models made from inexpensive materials like cardboard boxes and graspers to learn three-dimensional techniques such as laparoscopic suturing and knot tying. Simulation has been shown to be particularly useful during the Covid-19 pandemic to augment training since elective operative volume has decreased in almost every country worldwide.

While telehealth in LMICs has made several promising advances, its full potential has yet to be realized. Poor internet connectivity threatens to limit its usefulness especially in rural areas, which stand to benefit the most from these technologies. The use of tools such as mobile phones and mHealth apps will probably be more sustainable than ones requiring high bandwidth and expensive equipment. The expanded access made possible by 3G- enabled devices offers potential solutions to the challenges of internet bandwidth. Simple technologies such as the instant messaging platform like WhatsApp have been successfully used for consultation. In addition, metadata from these platforms can be used for quality improvement such as tracking the number of patients requiring follow-up or further work-up at higher level hospitals.

In conclusion, innovation in telehealth has the potential to improve access to timely and quality surgical care by mitigating barriers to referrals, and improving training, and mentorship. The Covid-19 pandemic, which has limited patient and provider mobility, has forced beta-testing of many of these new platforms. Given the necessity for telehealth in many LMICs, the effectiveness of these innovations must be urgently investigated especially in settings where limited resources can make translation to real-world solutions a challenge.

\section{References}

1. GlobalSurg Collaborative (2016) Mortality of emergency abdominal surgery in high-, middle- and low-income countries. Br J Surg 103:971-988

2. Fletcher RR, Olubeko O, Sonthalia H et al (2019) Application of machine learning to prediction of surgical site infection. Annu Int Conf IEEE Eng Med Biol Soc 2019:2234-2237

3. Morkel RW, Mann TN, du Preez G et al (2019) Orthopaedic referrals using a smartphone app: uptake, response times and outcome. S Afr Med J 109(11):859-864

4. Louis JS, Bukowski A, Rodrigues AN et al (2017) Global Cancer Institute multidisciplinary tumor boards as a tool to improve patterns of clinical practice for breast and gynecologic cancer in resource-limited settings. J Clin Oncol 35:6556-6556

5. Nieto-Calvache AJ, Zambrano MA, Herrera NA et al (2021) Resective-reconstructive treatment of abnormally invasive placenta: Inter Institutional Collaboration by telemedicine (eHealth). J Matern Fetal Neonatal Med 34:765-773

Publisher's Note Springer Nature remains neutral with regard to jurisdictional claims in published maps and institutional affiliations. 